\title{
Measurement of the Inclusive ep Scattering Cross Section at Low $Q^{2}$ and $x$ at $\mathrm{H} 1$
}

\author{
Alexandre Glazov*† \\ E-mail: glazov@mail.desy.de
}

\begin{abstract}
A measurement of the inclusive $e p$ scattering cross section is presented in the region of low momentum transfers, $0.2 \mathrm{GeV}^{2} \leq Q^{2} \leq 12 \mathrm{GeV}^{2}$, and low Bjorken $x, 5 \cdot 10^{-6} \lesssim x \lesssim 0.02$. The result is based on two data sets collected in dedicated runs by the H1 Collaboration at HERA at beam energies of $27.6 \mathrm{GeV}$ and $920 \mathrm{GeV}$ for positrons and protons in the years 1999 and 2000. A combination with data previously published by $\mathrm{H} 1$ leads to a cross section measurement of a few percent accuracy. The data are compared with theoretical models which apply to the transition region from photoproduction to deep inelastic scattering.
\end{abstract}

XVIII International Workshop on Deep-Inelastic Scattering and Related Subjects April 19 -23, 2010

Convitto della Calza, Firenze, Italy

\footnotetext{
* Speaker.

${ }^{\dagger}$ For the H1 collaboration
} 
Deep inelastic scattering (DIS) plays important role for the understanding of the proton structure and of the dynamics of parton interactions. The HERA collider operating at the high energy frontier using protons with energy of $E_{p}=920 \mathrm{GeV}$ and leptons (electrons and positrons) with energy of $E_{p}=27.5 \mathrm{GeV}$ extended tremendously the kinematic range compared to the previous fixed target experiments. The specially interesting kinematic range for the study of proton dynamics is at low values of Bjorken- $x$ and the absolute four momentum transfer $Q^{2}$. This interest arises because of the interplay between hard, perturbative calculable and soft partonic dynamics, combined with a high partonic density. For the HERA centre-of-mass energy squared $S=4 E_{p} E_{e}$, very low $x$ values of $x \sim 10^{-5}$ are achievable for $Q^{2}=1 \mathrm{GeV}^{2}$.

At low $Q^{2}$, the inclusive double differential neutral current scattering cross section is determined by the two structure functions $F_{2}$ and $F_{L}$. Excluding kinematic factors, the cross section in reduced form is

$$
\sigma_{r}\left(x, Q^{2}\right)=F_{2}\left(x, Q^{2}\right)-\frac{y}{1+(1-y)^{2}} F_{L}\left(x, Q^{2}\right) .
$$

Here inelasticity $y$ is given by $y=Q^{2} /(S x)$. The structure functions are related to the scattering cross sections for the transversely and longitudinally polarised photons, $\sigma_{T}$ and $\sigma_{L}$, as

$$
F_{2}=\frac{Q^{2}}{4 \pi^{2} \alpha}(1-x)\left(\sigma_{T}+\sigma_{L}\right), \quad F_{L}=\frac{Q^{2}}{4 \pi^{2} \alpha}(1-x) \sigma_{L} .
$$

In leading order QCD, the structure function $F_{2}$ determines the quark parton densities and $F_{L}$ vanishes. At higher orders, $F_{L}$ is predominantly determined by the gluon density. Experimentally, the structure function $F_{L}$ is much harder to measure because of the kinematic suppression: it contributes significantly only for large values of $y$.

The $\mathrm{H} 1$ collaboration performed several measurements and phenomenological analyses of the proton structure functions. The results based on the data collected in the years 1999-2000 in $e^{+} p$ scattering mode and combination of those with previous measurements are reported in [1]. This note is a short summary of this analysis.

A low $Q^{2}$ DIS event reconstructed in the H1 detector is shown in figure 1 . Since both the scattered electron and hadronic final state are measured in the H1 detector, several methods for kinematic reconstruction are available. For this low $Q^{2}$ analysis, the $\mathrm{H} 1$ collaboration uses information solely from the scattered electron (its energy $E_{e}^{\prime}$ and scattering angle $\theta_{e}$ ) for high $y \geq 0.1$ and combines this information with hadrons information for low $y<0.1$. The separation in $y$ is based on the resolution of the methods. Usage of the hadronic final state for event kinematics reconstruction allows to measure an effective lepton beam energy on event by event basis. This also allows background suppression and extends the kinematic reach by using events with large initial state QED radiation, for which the effective centre-of-mass energy is reduced.

Measuring at low $Q^{2}$ requires detection of the scattered lepton at $\theta_{e} \sim 180^{\circ}$ which corresponds to low radii in the SpaCal calorimeter. Dedicated runs were performed in the year 1999 to enable efficient triggers in this region allowing measurements up to $\theta_{e}=176.5^{\circ}$. In addition, special HERA run with the vertex position shifted to $Z=+70 \mathrm{~cm}$ was taken in the year 2000, which gives access to angles $\theta_{e}=178^{\circ}$.

At low $Q^{2}$, the DIS scattering cross section is very high and precision is mainly determined by the systematic uncertainties. A detailed study of the electromagnetic scale allowed a reduction 
of the scale uncertainty $\delta E_{e}^{\prime} / E_{e}^{\prime}$ to $0.2 \%$ at $E_{e}^{\prime}=27.5 \mathrm{GeV}$ to be made. The detector alignment is understood to better than $0.2 \mathrm{mrad}$, owing to high precision measurement of $\theta_{e}$ by the BST. The main source of uncertainty arises from the electron track reconstruction which amounts to $2 \%$ uncertainty in the cross section.

The accuracy of the 1999-2000 data are of comparable precision with the data collected by the $\mathrm{H} 1$ collaboration in 1995 and 1996 using $E_{p}=820 \mathrm{GeV}$ [2]. An averaging procedure [3] has been developed to combine these data sets which takes into account correlated systematic uncertainties. The combined data reach $1.7 \%$ accuracy for the bulk of the phase space.

The resulting combined cross section is shown in figure 2 as a function of $x$ for different $Q^{2}$ bins. For each $Q^{2}$ bin, the cross section increases as $x \rightarrow 0$. This increase is tamed somewhat at lowest $x$ values which can be atributed to the influence of the structure function $F_{L}$, see equation 1 . The data are compared to a " $\lambda$ fit" peformed for each $Q^{2}$ bin. In this fit the cross section is parameterised as

$$
\sigma_{r}=c x^{-\lambda}\left[1-\frac{y^{2}}{1+(1-y)^{2}} \frac{R}{R+1}\right]
$$

where $c, \lambda$ and $R$ are free parameters for each $Q^{2}$ bin. From these fits, the values of $\lambda$ are approximately constant $\lambda \approx 0.15$ for $Q^{2} \leq 2 \mathrm{GeV}^{2}$ and rise linearly for $Q^{2} \geq 2 \mathrm{GeV}^{2}$, as expected from the QCD evolution. The fit within this model yields a $Q^{2}$ independent large value of $R \approx 0.5$.

The data are also analysed in terms of fractal [4] and dipole models [5, 6]. Results of this analysis are presented in figure 3 which compares the $\mathrm{H} 1$ data represented as virtual photon-proton

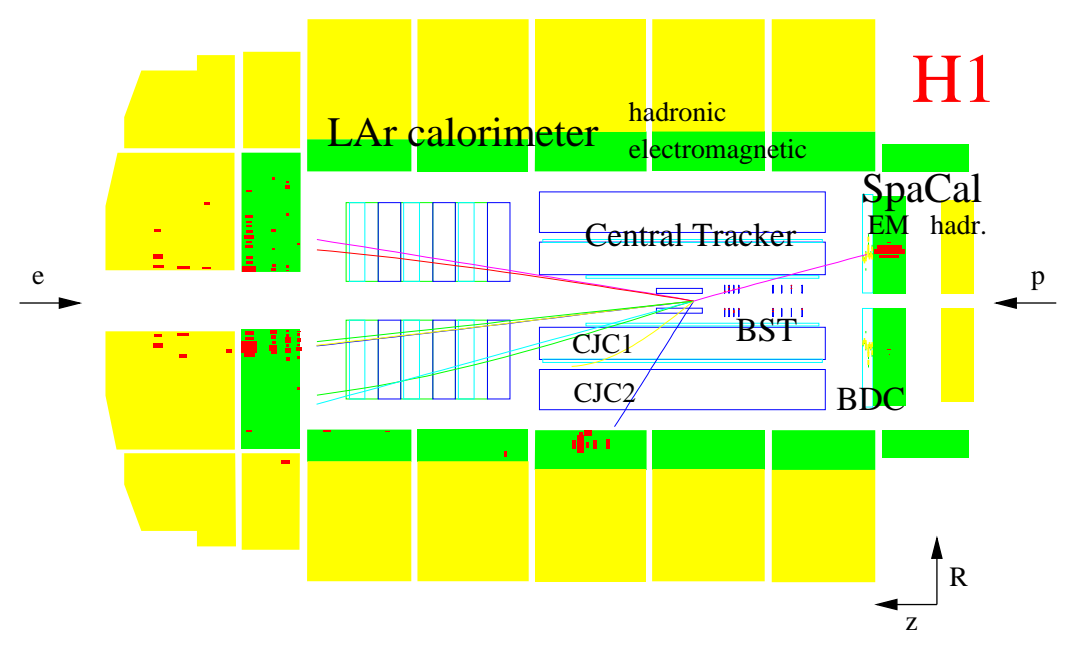

Figure 1: A low $Q^{2}$ event as reconstructed in the H1 detector. The positron and proton beam directions are indicated by the arrows. For the coordinate system used at HERA, the $z$ axis points in the direction of the proton beam. The nominal interaction point position corresponds to $Z=0$. The interaction vertex is reconstructed using the hadronic final state tracks in the central tracker (lines pointing towards proton beam direction) and the scattered positron (backward pointing line) in the backward tracker. The central tracker consists of (from the beam line outwards) the silicon tracker, the drift chambers CJC1 and CJC2, it is surrounded by the liquid argon (LAr) calorimeter. The detector operates in a solenoidal magnetic field of 1.16 T. The positron trajectory is reconstructed in the Backward Silicon Tracker (BST) and in the Backward Drift Chamber (BDC). The positron energy is determined using the SpaCal calorimeter. The hadronic final state is detected in the central and forward tracking detectors, and in the LAr calorimeter. 


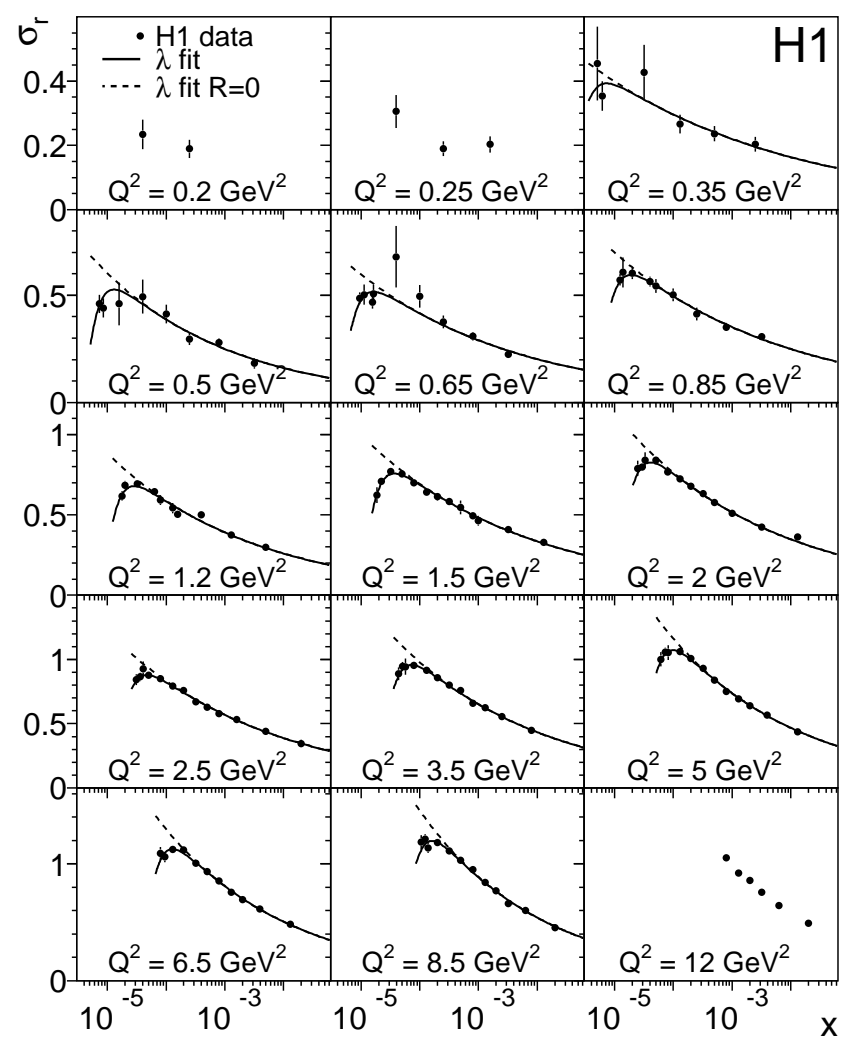

Figure 2: Reduced cross section $\sigma_{r}$, from the low $Q^{2} \mathrm{H} 1$ data, as a function of $x$ compared to the $\lambda$ fit result (solid line) and to a $\lambda$ parameterisation with the same values of $c\left(Q^{2}\right)$ and $\lambda\left(Q^{2}\right)$ but $R=0$ (dashed line). The errors represent the statistical and systematic uncertainties added in quadrature.

scattering cross section

$$
\sigma_{\gamma^{*} p}^{\mathrm{eff}}=\sigma_{T}+\left[1-\frac{y^{2}}{1+(1-y)^{2}}\right] \sigma_{L}
$$

with ZEUS data at lower [7] and higher $Q^{2}$ [8] and with the fits. All models can describe the data with similar $\chi^{2} / n_{d f}$. For the fractal model fit, which does not predict $F_{L}$ from first principles, $F_{L}$ is calculated assuming constant $R$ and a large value of $R=0.56 \pm 0.07$ is obtained. The dipole models predict both $F_{2}$ and $F_{L}$ structure functions. The structure function $F_{L}$ obtained from the dipole model fits corresponds approximately to $R \approx 0.25$. Good quality of the data description by the models with very different $R$ can be understood by comparing $F_{2}$ from them. The dipole models include saturation effects and predict a taming of the $F_{2}$ rise as $x \rightarrow 0$ compared to the $\lambda$ fit and the fractal model. The low value of $R \approx 0.25$ is consistent with the recent direct measurement of $F_{L}$ performed by the $\mathrm{H} 1$ collaboration at medium $Q^{2}$ [9].

To summarise, a new measurement of the inclusive DIS cross section has been published by the $\mathrm{H} 1$ collaboration for low $0.2 \leq Q^{2} \leq 12 \mathrm{GeV}^{2}$. These data are compared to phenomenological models and are also included in QCD fits [10] as well as in the combination with the ZEUS data [11], leading to a new level of precision in understanding of parton dynamics at low $x$. 


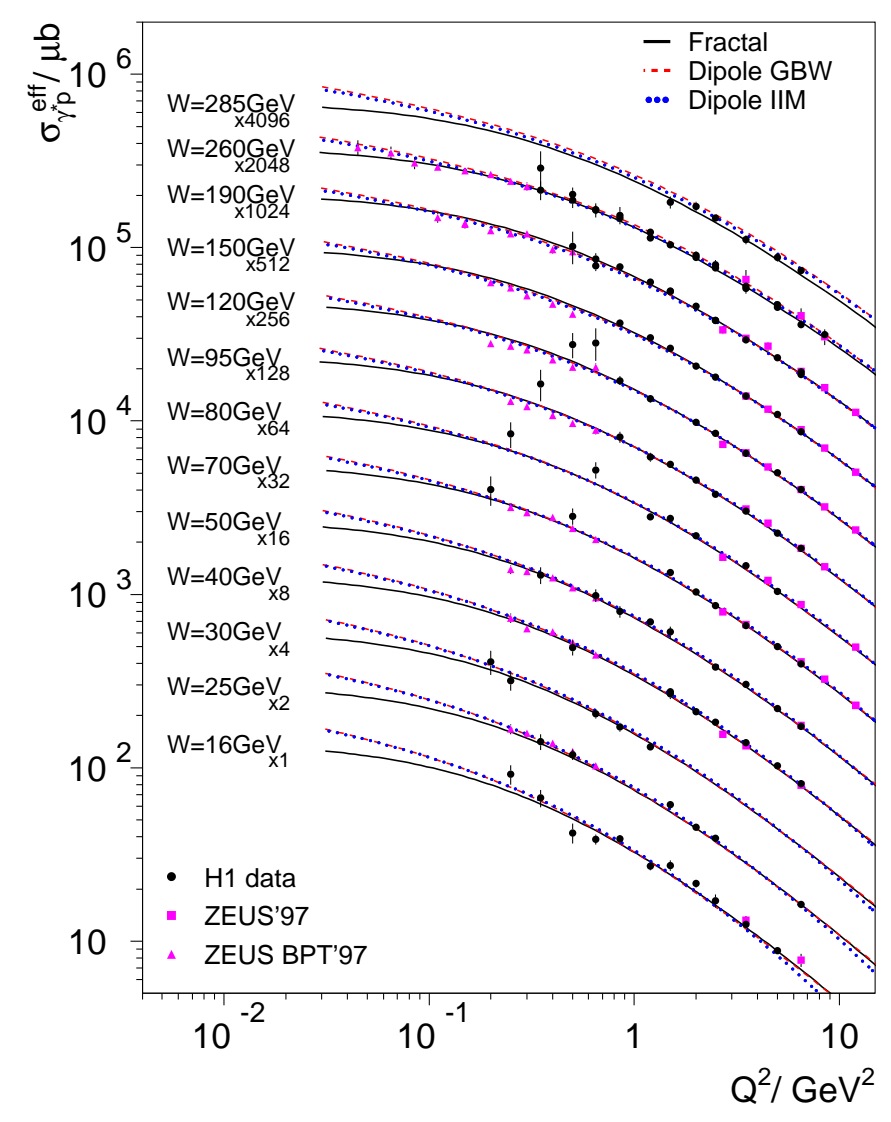

Figure 3: Measurement of the virtual photon-proton cross section $\sigma_{\gamma^{*} p}^{\text {eff }}$ as a function of $Q^{2}$ at various values of $W$. The cross sections for different $W$ values are multiplied with the factors indicated in the figure. The errors represent the statistical and systematic errors added in quadrature. The H1 results are compared to data obtained by the ZEUS experiment and to the fractal and dipole model fit results.

\section{References}

[1] F.D. Aaron et al. [H1 Collaboration] Eur.Phys.J. C63:625-678, (2009).

[2] C. Adloff et al. [H1 Collaboration], Nucl. Phys. B 497, 3 (1997);

C. Adloff et al. [H1 Collaboration], Eur. Phys. J. C 21, 33 (2001).

[3] A. Glazov, AIP Conf. Proc. 792, 237 (2005).

[4] T. Laštovička, Eur. Phys. J. C 24, 529 (2002).

[5] K. Golec-Biernat and M. Wüsthoff, Phys. Rev. D 59, 014017 (1999).

[6] E. Iancu, K. Itakura, and S. Munier, Phys. Lett. B590, 199 (2004).

[7] J. Breitweg et al. [ZEUS Collaboration], Phys. Lett. B487, 53 (2000).

[8] S. Chekanov et al. [ZEUS Collaboration], Eur. Phys. J. C 21, 443 (2001).

[9] F.D.Aaron et al. [H1 Collaboration], Phys. Lett. B665, 139 (2008).

[10] F.D.Aaron et al. [H1 Collaboration], Eur. Phys. J. C 64, 561 (2009).

[11] F.D.Aaron et al. [H1 and ZEUS Collaborations], JHEP 1001, (2010). 